\title{
Modeling and Simulation of Phase Equilibrium in Dynamic Systems
}

\author{
T. DAHL $\dagger$, A. HASHEMI-AHMADY $\ddagger$ and B. LIE* $\ddagger$
}

Keywords: Process dynamics, phase equilibrium, process simulation, numerical methods.

\begin{abstract}
The article reports an investigation on how to efficiently describe phase equilibrium in dynamic systems, and how to solve the resulting equations numerically. An overview of possible solution strategies is given. Different thermodynamic models are briefly reviewed, and important algorithms like PT flash are described. A flash tank with a mixture of propane and propylene is simulated to illustrate some different solution strategies.
\end{abstract}

\section{Introduction}

Thermodynamics is a mature theoretical field, and the last 25 years have seen a rapid progress in thermodynamic models enabling realistic computer simulations. The theory is traditionally rooted in steady state thinking (Denn, 1987), yet dynamic simulation studies are reported (e.g., Gani et al., 1991). During the last 10 years, process simulators which solve dynamic equilibrium problems (e.g., SPEEDUP, 1993; HYSYS, 1995) have emerged.

It is thus important to have a good overview of how such computations are performed in order to be able to assess the (often implicit) assumptions that are introduced, and the quality of the results. Also, it is important to have an idea of what part of the computations may fail, and why they may fail. This will make it possible to check out alternative algorithms. Furthermore, dynamic phase equilibrium simulations are increasingly used in control and optimization studies. It is thus important to develop ever more efficient simulation algorithms.

This article gives a brief survey of how to formulate models describing dynamics of mass and energy in systems with phase equilibrium. Algorithms for solving the resulting set of equations are discussed. The article is organized as follows. In section 2 , an overview of phase equilibrium in dynamic systems is given, as well as possible solution strategies. In section 3, a brief survey of thermodynamics is given, including equilibrim conditions. In section 4 , models necessary for thermodynamic calculations are surveyed. In section 5, some standard algorithms for doing thermodynamic calculations are discussed. In section 6, a few simulation results are presented, and finally, in section 7, some conclusions are drawn.

Received 23 September 1996.

An early version of this paper was presented at the SIMS '96 Applied Modelling and Simulation Conference, Trondheim, Norway, June 1996.

$\dagger$ Statoil Research Department, Trondheim.

$\ddagger$ Telemark College, N-3914 Porsgrunn, Norway.

* Author to whom correspondence should be addressed. 


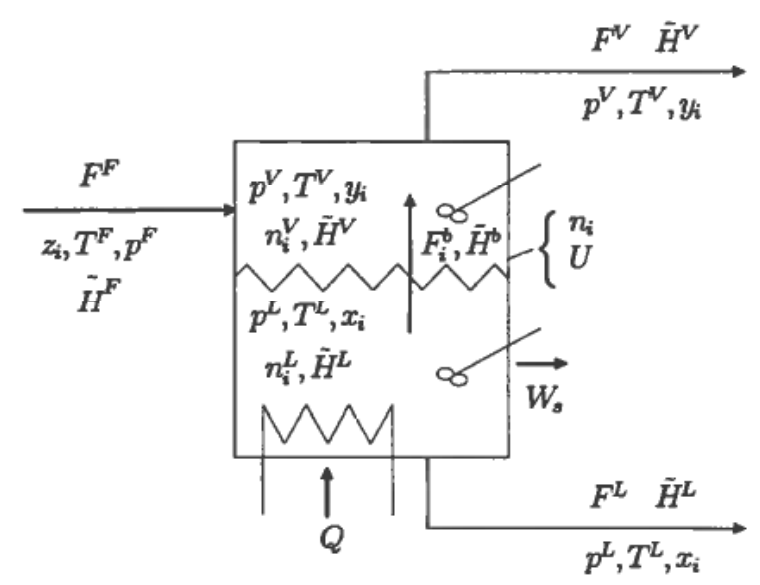

Figure 1. Sketch of a continuous flash tank.

\section{Overview: phase equilibrium in dynamic systems}

\subsection{Material and energy balance}

When the two phases of Fig. 1 are at equilibrium, such a system is denoted a flash tank (Smith and Van Ness, 1987). The total volume of the system is $V$. Superscript $F$ denotes feed, superscript $V$ denotes vapor, and superscript $L$ denotes liquid. $F$ is molar flow rate, $T$ is temperature, $p$ is pressure, $n_{i}$ is the total number of moles of component $i$ in volume $V$, while $n_{i}^{V}$ and $n_{i}^{L}$ are the number of moles of component $i$ in the vapor phase and liquid phase, respectively. $z_{i}$ is the mole fraction of component $i$ in the feed, $y_{i}$ is the mole fraction of $i$ in the vapor phase, and $x_{i}$ is the mole fraction of $i$ in the liquid phase. $\tilde{H}$ denotes molar enthalpy, and $U$ is internal energy. $Q$ is heat added to volume $V$, while $W_{s}$ is some work removed from the system.

In order to make the presentation less abstract, assume that the system in Fig. 1 is a binary mixture. Then material balances for the system can be expressed as

$$
\begin{aligned}
& \frac{d n_{1}}{d t}=F^{F} z_{1}-F^{V} y_{1}-F^{L} x_{1}=f_{n_{1}} \\
& \frac{d n_{2}}{d t}=F^{F} z_{2}-F^{V} y_{2}-F^{L} x_{2}=f_{n_{2}} .
\end{aligned}
$$

If the mixture consisted of $m$ components, the material balances would consist of $m$ equations $d n_{i} / d t=F^{F} z_{i}-F^{v} y_{i}-F^{L} x_{i}, i \in\{1, \ldots, m\}$ instead of the two equations in eqs. 1-2. If a reaction takes place, rates of reaction must be included in the material balances.

When neglecting kinetic and potential energy, the energy balance becomes

$$
\frac{d U}{d t}=F^{F} \tilde{H}^{F}-F^{v} \tilde{H}^{V}-F^{L} \tilde{H}^{L}+Q-W_{s}=f_{U} .
$$

The molar enthalpies are complicated functions of pressure, temperature, and mole fractions; $\tilde{H}^{V}=\tilde{H}^{V}\left(p^{V}, T^{V}, y_{1}, y_{2}\right)$ while $\tilde{H}^{L}=\tilde{H}^{L}\left(p^{L}, T^{L}, x_{1}, x_{2}\right)$.

The total internal energy is $U=\tilde{H}^{V} n^{V}+\tilde{H}^{L} n^{L}-p V$, where $n^{V}=\sum_{i=1}^{m} n_{i}^{V}$ and $\sum_{i=1}^{m} n_{i}^{L}$; hence for a binary mixture $U=U\left(p^{V}, p^{L}, T^{V}, T^{L}, y_{1}, y_{2}, x_{1}, x_{2}\right)$. 


\subsection{Equilibrium conditions}

When the system in Fig. 1 is at equilibrium, each phase is homogeneous throughout, and

$$
\begin{aligned}
& p^{V}=p^{L} \\
& T^{V}=T^{L} \\
& f_{i}^{V}=f_{i}^{L}, i \in\{1, \ldots, m\}
\end{aligned}
$$

where $f_{i}^{i}$ is the fugacity of component $i$ in phase $j \in\{\mathrm{V}, L\}$. The fugacity is a complex function of, e.g., pressure, temperature, and mole fraction of the different components.

In conclusion, the equilibrium conditions represent a number of nonlinear algebraic equations that must be satisfied while solving the dynamic model given by material and energy balances. Such problems are denoted Differential Algebraic Equations (DAE) (Brenan et al., 1989).

\subsection{Simulation strategies}

There are several possible strategies for solving the DAEs in the dynamic phase equilibrium model. To illustrate the methods, consider a binary mixture.

1. Straightforward UV flash calculation.

(a) Specify the initial values (e.g., at $t=0$ ) of $n_{1}, n_{2}$, and $U$. Solve algebraic equations to yield $p, T, y_{i}, x_{i}$, etc. at initial time. This is known as an $U V$ flash calculation, and it usually involves solving implicit algebraic equations iteratively.

(b) With $p, T, y_{i}, x_{i}$, etc., known, the vector fields $f_{n 1}, f_{n 2}$, and $f_{U}$ in eqs. 1,2 , and 3 can be computed.

(c) Finally, the states $n_{1}, n_{2}$, and $U$ can be computed for the next discretization point using, e.g. Runge-Kutta methods.

2. Use of DAE solver. Discretization methods for solving DAEs have been developed which only require the user to specify the differential and algebraic equations (Brenan et al., 1989); these are of form $F(x, d x / d t, t)=0$. The discretization methods are known as DAE solvers. Different levels of structuring of the DAEs (Ramirez, 1989) can be used to help the DAE solvers succeed.

3. Combined PT flash and UV flash calculations. In practice, most people find it hard to interpret energy. It is easier to relate to, e.g., pressure $p$, temperature $T$, and overall mole fraction $\chi_{i}=n_{i} / n$ of component $i$. Thus, assume that $p, T$, and $\chi_{1}$ are specified for the volume $V$ at initial time.

(a) When $p, T$, and $\chi_{1}$ are specified for a fixed volume $V$, all remaining quantities like $y_{i}, x_{i}, n_{i}$, etc., can be computed. This computation is known as a PT flash, and it usually involves solving implicit algebraic equations iteratively.

(b) With $p, T, y_{i}, x_{i}$, etc., known, the vector fields $f_{n 1}, f_{n 2}$, and $f_{U}$ in eqs. 1,2 , and 3 can be computed.

(c) The states $n_{1}, n_{2}$, and $U$ can be computed for the next discretization point using, e.g., Runge-Kutta methods.

(d) Finally, perform a UV flash calculation to compute $p, T$, and $\chi_{1}$ at the new point in time.

4. Avoiding UV flash calculations by transformation of differential variables. 
(a) Assume that $p, T$, and $\chi_{1}$ are specified for the volume $V$ at initial time.

(b) With $p, T$, and $\chi_{1}$ known at a given point in time, it is possible to compute $n_{1}$, $n_{2}$, and $U$ at the same point in time. Hence, $n_{1}=n_{1}\left(p, T, \chi_{1}\right), n_{2}=n_{2}\left(p, T, \chi_{1}\right)$, and $U=U\left(p, T, \chi_{1}\right)$. Thus

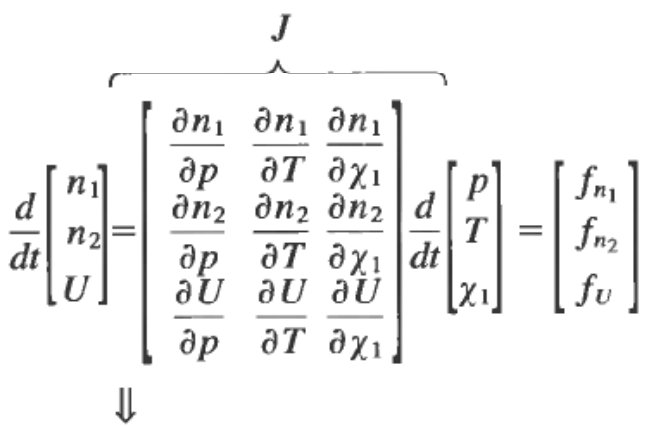

$$
\begin{aligned}
& \frac{d}{d t}\left[\begin{array}{l}
p \\
T \\
\chi_{1}
\end{array}\right]=J^{-1}\left[\begin{array}{l}
f_{n_{1}} \\
f_{n_{2}} \\
f_{U}
\end{array}\right]=\left[\begin{array}{l}
f_{p} \\
f_{T} \\
f_{\chi_{1}}
\end{array}\right]
\end{aligned}
$$

where $J$ is the Jacobian from $\left(p, T, \chi_{1}\right)$ to $\left(n_{1}, n_{2}, U\right)$.

The Jacobian $J$ may be computed using numerical differentiation (Gill et al., 1981), or developing exact symbolic expressions for $J$ (these may include PT flash calculations). Symbolic expressions may be found by using, e.g., Maple (Char et al., 1992).

(c) Use PT flash calculations to compute $f_{n_{1}}, f_{n_{2}}$, and $f_{U}$.

(d) Finally, the states $p, T$, and $\chi_{1}$ can be computed for the next discretization point using Runge-Kutta methods, the Gear method, etc.

5. Avoiding both PT flash and UV flash calculations; akin to a method discussed in Bosley (1994).

(a) Assume that $p, T$, and $\chi_{1}$ at initial time are specified for the volume $V$.

(b) From off-line computations involving PT flash and UV flash calculations, fit simple, explicit correlations of sufficient accuracy for $n_{1}=n_{1}\left(p, T, \chi_{1}\right)$, $n_{2}=n_{2}\left(p, T, \chi_{1}\right), U=U\left(p, T, \chi_{1}\right), \tilde{H}^{V}=\tilde{H}^{V}\left(p, T, \chi_{1}\right)$, and $\tilde{H}^{L}\left(p, T, \chi_{1}\right)$.

(c) Finally, it is a simple task to develop an ODE for $p, T$, and $\chi_{1}$, confer eq. 7 , with explicit symbolic expressions for the Jacobian $J$ and $f_{n_{1}}, f_{n_{2}}$, and $f_{U}$.

\subsection{Modeling principles}

The index of a DAE system $F(x, \dot{x}, t)=0$ is unity if rank $\partial F / \partial \dot{x}=\operatorname{dim} x$. By differentiating the relevant algebraic equations in $F(x, \dot{x}, t)=0 \mathrm{wrt}$. time once and adding the resulting expressions to $F(x, \dot{x}, t)=0$, the new set of DAEs is $F_{1}(x, \dot{x}, t)=0$. This process can be repeated to yield $F_{j}(x, \dot{x}, t)=0$. The index $I$ of the original DAE $F(x, \dot{x}, t)=0$ is equal to the least number $j=I$ such that rank $\partial F_{I} / \partial \dot{x}=\operatorname{dim} x$. Higher index problems are DAEs with $I>1$. Such higher index problems are usually more difficult to solve than problems of index zero (ODEs) and unity, Brenan et al. (1989).

By formulating overall material and energy balances for the flash tank in conjunction with specifying algebraic equilibrium conditions as described in the previous sections, the resulting DAEs are of index 1 (Moe, 1995). 


\section{Equilibrium conditions}

The fundamental relations of thermodynamics may be developed from four postulates and the use of standard calculus (Callen, 1985). Although most authors instead prefer to trace the roots of these relations (Bejan, 1988; Modell and Reid, 1983), the presentation that follows is inspired by Callen's approach.

The entropy $S$ is a function of $U, V$, and $n_{1}, \ldots, n_{m}$, and attains its maximum at equilibrium. When the entropy function $S\left(U, V, n_{1}, \ldots, n_{m}\right)$ is known, any thermodynamic information can be extracted from this function; hence $S=S\left(U, V, n_{1}, \ldots, n_{m}\right)$ is known as a fundamental equation. As an alternative we can extract any information from $U\left(S, V, n_{1}, \ldots, n_{m}\right)$ where $U$ attains its minimum at equilibrium; $U=U\left(S, V, n_{1}, \ldots, n_{m}\right)$ is also a fundamental equation. Let $\left\{n_{i}\right\}$ denote $\left\{n_{1}, \ldots, n_{m}\right\}$ and $\left\{n_{i}\right\} \backslash n_{j}$ denote $\left\{n_{i}\right\}$ except $n_{j}$. As examples of what can be computed from a fundamental expression, the absolute temperature is $T \triangleq \partial U /\left.\partial S\right|_{V,\left\{n_{i}\right\}}=f\left(S, V,\left\{n_{i}\right\}\right)$, the pressure is $p \triangleq-\partial U /\left.\partial V\right|_{S,\left\{n_{i}\right\}}=f\left(S, V,\left\{n_{i}\right\}\right)$, while the (electro)chemical potential of component $j$ is $\mu_{j} \triangleq \partial U /\left.\partial n_{j}\right|_{s, V .\left\{n_{i} \backslash \backslash n_{j}\right.}=f(S$, $\left.V,\left\{n_{i}\right\}\right)$.

Conceptually, it is possible to solve, e.g., $T=T\left(S, V,\left\{n_{i}\right\}\right)$ for $S$ to yield $S=S(T$, $\left.V,\left\{n_{i}\right\}\right)$, and thus express $U$ as $U=U\left(T, V,\left\{n_{i}\right\}\right)$. This would, however, not be a fundamental equation any more; essentially, this is due to the loss of some integration constant in the definition of $T$.

Still, it is possible to change variables and retain a fundamental equation; this can be done via a Legendre transformation. The Legendre transformation leads to fundamental equations $H=U+p V=H\left(S, p,\left\{n_{i}\right\}\right), A=U-T S=A\left(T, V,\left\{n_{i}\right\}\right)$, and $G=H-T S=U+p V-T S=G\left(p, T,\left\{n_{i}\right\}\right)$ where $H$ is enthalpy, $A$ is Helmoltz' free energy, and $G$ is Gibb's free energy. It can be shown that equilibrium for composite systems is found at the minimum of, e.g., $U, A$, or $G$. Thus, $\mu_{i}$ can be expressed as

$$
\mu_{i}=\left.\frac{\partial U}{\partial n_{j}}\right|_{S, V,\left\{n_{i} \backslash \backslash n_{j}\right.}=\left.\frac{\partial G}{\partial n_{j}}\right|_{p, T,\left(n_{i}\right) \backslash n_{j}}=\left.\frac{\partial A}{\partial n_{j}}\right|_{T, V,\left\{n_{i} \backslash \backslash n_{j}\right.} .
$$

Since $p$ and $T$ are more tangible quantities than $S$, etc., the use of Gibb's free energy is popular in engineering literature. However, when computing $\mu_{i}$ from equations of state, the use of Helmholtz' energy has some advantages.

For a system composed of $\pi$ phases, it follows from the postulates and standard calculus of multivariable systems that in the equilibrium state

$$
\begin{aligned}
& T^{(1)}=T^{(2)}=\cdots=T^{(\pi)} \\
& p^{(1)}=p^{(2)}=\cdots=p^{(\pi)} \\
& \mu_{i}^{(1)}=\mu_{i}^{(2)}=\cdots=\mu_{i}^{(\pi)}
\end{aligned}
$$

where the superscript indicates the phase and subscript $i$ indicates component $i$.

Unfortunately, computing an absolute value for the chemical potential $\mu_{i}$ is, strictly speaking, not possible; it is only possible to compute $\mu_{i}$ relative to its value at a reference state. There are, however, two other obstacles which disable us to calculate the absolute value of the chemical potential. One is that the chemical potentials become negative infinite as the system pressure approaches zero. The other one is that the chemical potential of a component in a mixture also becomes negative infinite as the concentration of that component approaches zero, Modell and Reid (1983). 
To resolve these problems, it is common practice to introduce a monotonic mapping of the chemical potential to yield the fugacity $f_{i}$ (following Sandler, 1989)

$$
\mu_{i}-\mu_{i}^{\mathrm{ig}}=R T \ln \frac{f_{i}}{\zeta_{i} p}
$$

where superscript ig indicates ideal gas. It is useful to introduce the dimensionless fugacity coefficient $\varphi_{i}$, as

$$
\varphi_{i} \triangleq \frac{f_{i}}{\zeta_{i} p} \Rightarrow \mu_{i}-\mu_{i}^{\mathrm{ig}}=R T \ln \varphi_{i}
$$

By denoting $\mu_{i}\left(p^{0}, T^{0},\left\{\zeta_{i}^{0}\right\}\right)=\mu_{i}^{0}$ and $f_{i}\left(p^{0}, T^{0},\left\{\zeta_{i}^{0}\right\}\right)=f_{i}^{0}$ where superscript 0 indicates a particular choice of $\left(p, T,\left\{\zeta_{i}\right\}\right)$, from eq. 12 it follows that $\mu_{i}^{0}-\mu_{i}^{\mathrm{ig}}=R T \ln \left(f_{i}^{0} /\left(\zeta_{i} p\right)\right)$. Subtracting this expression from eq. 12 leads to

$$
\mu_{i}-\mu_{i}^{0}=R T \ln \frac{f_{i}}{f_{i}^{0}}
$$

Thus, (following Prausnitz et al., 1986) we could alternatively have defined fugacity from eq. 14 with the additional requirement that

$$
f_{i}^{\mathrm{g}}=\zeta_{i} p
$$

Inserting eq. 14 into the equilibrium condition in eq. 11 leads to a new condition for phase equilibrium

$$
f_{i}^{(1)}=f_{i}^{(2)}=\cdots=f_{i}^{(\pi)} .
$$

Application of eq. 16 to specific phase equilibrium problems requires the use of models of mixture behavior.

\section{Thermodynamic models}

In order to compute the equilibrium state in our model, we need to compute the fugacity of all phases for a given component. The fugacity is a relatively complicated function of, e.g., pressure, temperature, and composition. It is a standard trick in mathematics to relate an expression to a departure from a simpler expression, e.g., Taylor's theorem states that if $f$ has a continuous first derivative, then $f(x+h)-f(x)=f^{\prime}(\xi) h$ where $\xi \in[x, x+h]$. In practice, approximations to $f^{\prime}(\xi)$ are then used. In thermodynamics, it is common to devise a correlation for the departure from either an ideal gas or an ideal solution.

\subsection{Residual properties: departure from ideal gas}

Let $\tilde{M}$ be the molar value of any extensive ${ }^{1}$ thermodynamic property; the value for $\tilde{M}$ is $\tilde{M}=M / n$ where $M$ is the total value of the property and $n$ the number of moles in the mixture. Furthermore, define the partial molar value of component $i$ as

$$
\left.\bar{M}_{i} \triangleq \frac{\partial M}{\partial n_{i}}\right|_{p, T,\left\{n_{j} \backslash \backslash n_{i}\right.}
$$

\footnotetext{
' Extensive properties are homogeneous of degree 1, e.g., Bejan (1988). In this context, this means that their value increases linearly with the number of moles.
} 
It follows from Euler's theorem (Modell and Reid, 1983) that

$$
M=\sum_{i=1}^{m} n_{i} \bar{M}_{i}=n \tilde{M}
$$

Note that this expression is only valid when $M$ is a function of intensive variables $p$ and $T$ in addition to the extensive $n_{i}$ 's. As an example, from eq. 8,

Next, define the residual property $M^{\mathrm{R}}$ as

$$
\mu_{i}=\bar{G}_{i}=\left.\frac{\partial A}{\partial n_{j}}\right|_{T, V .\left\{n_{j}\right) \backslash n_{i}} \neq \bar{A}_{i} .
$$

$$
M^{\mathrm{R}} \triangleq M-M^{\mathrm{ig}}
$$

where superscript ig denotes ideal gas. Similar expressions are defined for $\tilde{M}$ and $\bar{M}_{\mathrm{i}}$.

By choosing the reference state equal to that of ideal gas, eq. 15 yields $f_{i}^{0}=\zeta_{i} p$. Introducing the fugacity coefficient $\varphi_{i}$ from eq. 13, eqs. 19 and 8 yield

$$
R T \ln \varphi_{i}=\mu_{i}^{\mathrm{R}}=\left.\frac{\partial G^{\mathrm{R}}}{\partial n_{i}}\right|_{p, T,\left[n_{j} \backslash \backslash n_{i}\right.}=\left.\frac{\partial A^{\mathrm{R}}}{\partial n_{i}}\right|_{T, V,\left\{n_{j} \backslash \backslash n_{i}\right.}
$$

From eq. 20 and a selected equation of state (see next section), it is possible to compute $\varphi_{i}$ (Prausnitz et al., 1986) which enables us to calculate $f_{i}=\zeta_{i} p \varphi_{i}$.

Next, since $R T \ln \varphi_{i}=\bar{G}_{i}^{\mathrm{R}}$, application of eq. 18 allows us to compute $G^{\mathrm{R}}\left(p, T,\left\{n_{i}\right\}\right)$. Since $G^{\mathrm{R}}$ is a fundamental equation in $p$ and $T$, any other residual property can be computed from this expression, e.g., $H^{\mathrm{R}}, U^{\mathrm{R}}$, and $S^{\mathrm{R}}$. In order to complete the calculations, we need to find $G^{\mathrm{ig}}, H^{\mathrm{ig}}, U^{\mathrm{ig}}$, and $S^{\mathrm{ig}}$. Expressions for these quantities are developed in Smith and Van Ness (1987). Basically, they are computed from an expression for the ideal gas heat capacity $c_{p}^{\mathrm{ig}}(T)$, together with the gas phase heat of formation at given temperature and pressure, see Reid et al. (1988).

\subsection{Equations of state}

The limitations in accuracy of the computed fugacity coefficients from residual thermodynamics lie entirely in the ability of the applied equation of state to describe intermolecular forces for the actual system (Prausnitz et al., 1986). Since the departure functions in the case of residual thermodynamics are related to ideal gas, it is obvious that relatively complicated equations of state are needed in order to describe liquids. It is not necessary to use the same equation of state for each phase in equilibrium calculations. The choice of equation of state depends on several factors, e.g., the temperature and pressure of the actual system, what accuracy is needed, what phase the system is in, whether it is necessary with a model that can represent both phases, etc.

In the theory of corresponding states, all substances fit into the same equation of state

$$
F\left(\tilde{V}_{r}, T_{r}, p_{r}\right)=0,
$$

where $\left(\tilde{V}_{r}, T_{r}, p_{r}\right)=\left(\tilde{V} / \tilde{V}_{c}, T / T_{c}, p / p_{c}\right), \tilde{V}$ is the molar volume, and $\left(\tilde{V}_{c}, T_{c}, p_{c}\right)$ specifies the critical point. The critical parameters vary from substance to substance, see, e.g., Reid et al. (1988). A number of (semi) empirical equations of state are based 


\begin{tabular}{|c|c|c|}
\hline Family of EOS & Applicability & Comment \\
\hline Ideal gas law & $\begin{array}{l}\text { Low pressure } \\
\text { mixtures of gas }\end{array}$ & $\begin{array}{l}\text { Vapor at low } \\
\text { pressure }\end{array}$ \\
\hline Cubic EOS & $\begin{array}{l}\text { Moderately high } \\
\text { pressure mixtures }\end{array}$ & $\begin{array}{l}\text { Gas (and } \\
\text { liquid) phase }\end{array}$ \\
\hline More complex EOSs & High pressure mixtures & Gas and liquid phase \\
\hline Virial EOS & $\begin{array}{l}\text { Modest deviation from } \\
\text { ideal gas law }\end{array}$ & $\begin{array}{l}\text { Gas phase } \\
\text { Series in } \tilde{V}^{-1} \text { or } p \text {, \# terms } \\
\text { determine accuracy }\end{array}$ \\
\hline
\end{tabular}

Table 1. Merits of Equations of State (EOS).

upon the theory of corresponding states, e.g., those of van der Waal, Redlich-Kwong, Peng-Robinson, Benedict-Webb-Rubin, Lee-Kesler, etc.

The virial equation of state can be developed from statistical mechanics, and thus has a theoretical foundation. In short, it describes deviation from the ideal gas law as a power series in either $\tilde{V}^{-1}$ or $p$.

Some common equations of state are reviewed in Table 1. Sandler (1994) discusses a number of equations of state.

We have already mentioned the ideal gas law

$$
z \triangleq \frac{p \tilde{V}}{R T}=1 \text {, }
$$

where $z$ is known as the compressibility factor. Cubic equations of state constitute one family of equations, where "cubic" indicates that they can be written as a cubic polynomial in either $\tilde{V}$ or $z$. Consider a pure fluid with critical temperature $T_{c . i}$, critical pressure $P_{c, i}$, and acentric factor $\omega_{i}$ (instead of using $\tilde{V}_{c}$ ). The pure fluid Soave-Redlich-Kwong (SRK) equation is (Gmehling and Kolbe, 1988)

$$
p=\frac{R T}{\tilde{V}-b_{i}}-\frac{a_{i}}{\tilde{V}\left(\tilde{V}+b_{i}\right)}
$$

where $p$ is the pressure, $T$ is the temperature, $\tilde{V}$ is the molar volume of the pure fluid, and $R$ is the gas constant. $b_{i}$ and $a_{i}$ are functions of the critical parameters. In addition, $a_{i}$ is a function of temperature.

When considering a mixture of $m$ components, each with a mole fraction of $\zeta_{i}\left(x_{i}\right.$ for a liquid, $y_{i}$ for a vapor), it is common practice to postulate a pseudo fluid (one-fluid theory) satisfying, e.g., the SRK equation with parameters $a_{M}$ and $b_{M}$

$$
p=\frac{R T}{\tilde{V}-b_{M}}-\frac{a_{M}}{\tilde{V}\left(\tilde{V}+b_{M}\right)} .
$$

Parameters $a_{M}$ and $b_{M}$ are mixture parameters, and they are expressed as combinations of the pure fluid $a_{i}$ 's and $b_{i}$ 's. A common mixing rule is

$$
a_{M}=\sum_{i=1}^{m} \sum_{j=1}^{m} \zeta_{i} a_{i j} \zeta_{j}, \quad b_{M}=\sum_{i=1}^{m} \zeta_{i} b_{i}
$$

where $a_{i j}$ is given as $a_{i j}=\left(1-k_{i j}\right) \sqrt{a_{i} a_{j}}$. Here, $k_{i j}$ is a binary interaction parameter which is small for many mixtures, and $k_{i i} \equiv 0$. In the one component case, $a_{M}=a_{i}$ and $b_{M}=b_{i}$. 


\subsection{Residual properties from the SRK}

When an equation of state has been chosen, an expression for the fugacity coefficient can be computed from eq. 20. This leads to expressions for $f_{i}$ and $G^{\mathrm{R}}$. From the expression for $G^{\mathrm{R}}$ and ideal gas properties, it is possible to compute $G, H, U$, and $S$.

Gmehling and Kolbe (1988) list expressions for $\ln \varphi_{i}, \tilde{G}^{\mathrm{R}}, \tilde{H}^{\mathrm{R}}$, and $\tilde{S}^{\mathrm{R}}$ when the SRK equation of state is used. From these expressions and similar expressions for the ideal gas (Smith and Van Ness, 1987), expressions for $f_{i}, G, H, U$, and $S$ can thus easily be found.

When computing fugacity coefficients, it is necessary to find the molar volume of the vapor and liquid through solving the equation of state that is used. Gundersen (1982) discusses some problems that may occur when computing these molar volumes from cubic equations of state.

\subsection{Excess properties: departure from ideal solution}

In a previous section, the fugacity of a state has been computed based upon correlations for the departure of that state from the ideal gas state $\left(R T \ln \varphi_{i}=\mu_{i}^{\mathrm{R}}=\mu_{i}-\mu_{i}^{\mathrm{ig}}\right)$. Obviously, if the state of the system is a condensed phase (e.g., liquid phase), $\mu_{i}^{\mathrm{R}}$ is "large" and it is hard to find good correlations for this departure.

It thus makes sense to operate with the departure from some ideal liquid mixture state instead of the departure from the ideal gas state. When the reference state in eq. 14 is an ideal solution (is), the departure property is denoted an excess property $M^{\mathrm{E}}$

$$
M^{\mathrm{E}} \triangleq M-M^{\text {is }} \text {. }
$$

A mixture of components is denoted an ideal solution when the fugacity $f_{i}^{\text {is }}$ of the mixture at given $\left(p, T,\left\{\zeta_{i}\right\}\right)$ satisfies

$$
f_{i}^{\text {is } \triangleq} \zeta_{i} \hat{f}_{i}
$$

where $\hat{f}_{i}$ is the fugacity of pure component $i$ at a given $p$ and $T$. Thus, $f_{i}^{\text {is }}=\zeta_{i} \hat{f}_{i}(p, T)$.

The excess chemical potential is thus

$$
\mu_{i}^{\mathrm{E}} \triangleq \mu_{i}-\mu_{i}^{\mathrm{is}}=R T \ln \frac{f_{i}}{f_{i}^{\mathrm{s}}}=R T \ln \frac{f_{i}}{\zeta_{i} \hat{f}_{i}}=R T \ln \gamma_{i},
$$

where the activity coefficient $\gamma_{i}$ has been introduced. Thus

$$
R T \ln \gamma_{i}=\mu_{i}^{\mathrm{E}}=\left.\frac{\partial G^{\mathrm{E}}}{\partial n_{i}}\right|_{p, T,\left\{n_{j} \backslash \backslash n_{i}\right.}=\left.\frac{\partial A^{\mathrm{E}}}{\partial n_{i}}\right|_{T, V,\left\{n_{j} \backslash \backslash n_{i}\right.} .
$$

The parallel between the activity coefficient in eq. 22 and the fugacity coefficient in eq. 13 is apparent, as is the parallel between eqs. 23 and 20 .

Since $\mu_{i}^{\mathrm{E}}$ is identical to the partial molar excess Gibb's energy, $\mu_{i}^{\mathrm{E}}=\bar{G}^{\mathrm{E}}$, the activity coefficient is found from

$$
R T \ln \gamma_{i}=\left.\bar{G}^{\mathrm{E}} \triangleq \frac{\partial\left(n \tilde{G}^{\mathrm{E}}\right)}{\partial n_{i}}\right|_{p, T,\left\{n_{j} \backslash \backslash n_{i}\right.} .
$$

In the next section, we will consider some activity coefficient models which makes 
it possible to find expressions for $\gamma_{i}$. When we thus have an expression for $\gamma_{i}$, the fugacity is given as $f_{i}=\gamma_{i} f_{i}^{\text {is }}$.

From eq. $21, f_{i}^{\text {is }}=\zeta_{i} \hat{f}_{i}$ where $\hat{f}_{i}=\hat{f}_{i}(p, T)$. The standard way of calculating the pure component quantity $\hat{f}_{i}$ is to write it as (Sandler, 1989)

$$
\hat{f}_{i}=\hat{f}_{i}\left(p_{i}^{\text {sat }}, T\right) \theta_{i}(p, T)=\hat{f}_{i}^{V}\left(p_{i}^{\text {sat }}, T\right) \theta_{i}=\hat{\varphi}_{i}^{Y \text {,sat }} p_{i}^{\text {sat }} \theta_{i}
$$

where $\theta_{i}$ is the Poynting pressure correction, and $p_{i}^{\text {sat }}$ is the saturation pressure at $T$ for pure component $i$. $\hat{\varphi}_{i}^{V \text {,sat }}$ can be computed with the methods of residual thermodynamics, and the Poynting correction $\theta_{i}$ is given from

$$
R T \ln \theta_{i}=\int_{p^{\operatorname{sat}(T)}}^{p} \tilde{V} d p .
$$

In practice, semi empirical correlations for molar excess Gibb's energy, $\tilde{G}^{\mathrm{E}}$, are designed. When $\tilde{G}^{\mathrm{E}}$ is thus given, the fundamental expression is $G^{\mathrm{E}}=n \tilde{\mathrm{G}}^{\mathrm{E}}$. Hence, from the expression for $G^{\mathrm{E}}$, any excess property can be found, e.g., $H^{\mathrm{E}}, U^{\mathrm{E}}$, and $S^{\mathrm{E}}$. To complete the calculation of extensive properties, we need expressions for ideal solution $H^{\text {is }}, U^{\text {is }}$, and $S^{\text {is. }}$. These are developed in Smith and Van Ness (1987).

\subsection{Activity coefficient models}

Traditionally the excess molar Gibb's energy has been expressed as empirical power series, e.g., the Margules equations which are power series in mole fractions.

Modern theoretical developments in the molecular thermodynamics of liquid solution behavior are, however, based on the concept of local compositions. Within a liquid solution, local compositions are different from the overall mixture composition, and are presumed to account for the short-range order and nonrandom molecular orientation that results from differences in molecular size and intermolecular forces. The semi empirical equations Wilson, NRTL and UNIQUAC are all based on this concept. As an example, the Wilson equation for molar Gibb's energy of a binary mixture is

$$
\frac{\tilde{G}^{\mathrm{E}}}{R T}=-x_{1} \ln \left(x_{1}+\Lambda_{12} x_{2}\right)-x_{2} \ln \left(x_{2}+\Lambda_{21} x_{1}\right)
$$

with

$$
\begin{aligned}
& \Lambda_{12}=\frac{\tilde{V}_{2}}{\tilde{V}_{1}} \exp \left(-\frac{\lambda_{12}-\lambda_{11}}{R T}\right) \\
& \Lambda_{21}=\frac{\tilde{V}_{1}}{\tilde{V}_{2}} \exp \left(-\frac{\lambda_{12}-\lambda_{22}}{R T}\right) .
\end{aligned}
$$

Here, $\lambda_{i j}$ is the energy of interaction between molecules of component $i$ and $j$, and $\tilde{V}_{i}$ is the molar volume of pure liquid component $i$ at temperature $T$.

A further significant development based on the UNIQUAC equation, is the UNIFAC method in which activity coefficients are calculated from contributions of the various groups making up the molecules of a solution. The last decade has seen a nearly explosive development in the application of UNIFAC and other group contribution activity coefficient models, Sandler (1994).

Prausnitz et al. (1986) and Sandler (1994) give details of various activity coefficient models. Table 2 gives a brief account of some activity coefficient model families. 


\begin{tabular}{|c|c|}
\hline Activity coefficient model family & Applicability \\
\hline $\begin{array}{l}\text { Regular solution theory } \\
\text { (Scatchard-Hildebrand, van Laar etc.) }\end{array}$ & $\begin{array}{l}\text { Mixtures of nonpolar components } \\
\text { with similar intermolecular forces. }\end{array}$ \\
\hline $\begin{array}{l}\text { Athermal solution theory } \\
\text { (Flory and Huggins) }\end{array}$ & $\begin{array}{l}\text { Mixtures of components with similar } \\
\text { intermolecular forces, i.e. solutions } \\
\text { with only polar components or } \\
\text { only nonpolar components. }\end{array}$ \\
\hline Wilson's equation & $\begin{array}{l}\text { Mixtures of polar and nonpolar components. } \\
\text { Cannot describe liquid-liquid equilibrium. }\end{array}$ \\
\hline $\begin{array}{l}\text { Two-fluid theory } \\
\text { (UNIQUAC, NRTL, UNIFAC) }\end{array}$ & $\begin{array}{l}\text { Mixtures of both polar and } \\
\text { nonpolar components. }\end{array}$ \\
\hline Chemical theories & $\begin{array}{l}\text { Works best for solutions of polar } \\
\text { components and ionic systems. }\end{array}$ \\
\hline
\end{tabular}

Table 2. Merits of Activity Coefficient Models.

\subsection{Vapor-liquid equilibrium. An ideal system: Raoult's law}

At phase equilibrium between vapor phase and liquid phase, it is required that $f_{i}^{V}=f_{i}^{t}$. There is no requirement that the same equation of state is used when computing $f_{i}^{V}$ or $f_{i}^{L}$. In practice, it is even relatively common to use residual thermodynamics for the vapor phase and excess thermodynamics for the liquid phase.

For a general vapor-liquid equilibrium calculation using residual and excess thermodynamics,

$$
\begin{aligned}
& f_{i}^{V} \underset{\mathbb{}=}{=} f_{i}^{L} \\
& p y_{i} \varphi_{i}^{V}=\gamma_{i}^{L} x_{i} \hat{f}_{i}^{L} \\
& K_{i} \triangleq \frac{y_{i}}{x_{i}}=\gamma_{i}^{L} \frac{\hat{\varphi}_{i}^{V \text {,sat }}}{\varphi_{i}^{V}} \frac{p_{i}^{\text {sat }}}{p} \theta_{i}=\frac{\gamma_{i}^{L}\left(p, T,\left\{x_{i}\right\}\right) \hat{\varphi}_{i}^{V}\left(p_{i}^{\text {sat }}, T\right) p_{i}^{\text {sat }}(T) \theta_{i}(p, T)}{p \varphi_{i}^{V}\left(p, T,\left\{y_{i}\right\}\right)} .
\end{aligned}
$$

Here, $K_{i}$ is known as the $K$-value for component $i$.

Likewise, when residual thermodynamics is used for both phases, this yields the following $K$-value.

$$
K_{i}=\frac{\varphi_{i}^{L}}{\varphi_{i}^{V}}=\frac{\varphi_{i}^{L}\left(p, T,\left\{x_{i}\right\}\right)}{\varphi_{i}^{V}\left(p, T,\left\{y_{i}\right\}\right)} .
$$

When assuming that the vapor phase behaves like an ideal gas and the liquid phase behaves like an ideal solution, $f_{i}^{V}=f_{i}^{\mathrm{ig}}=y_{i} p$ and $f_{i}^{L}=f_{i}^{\mathrm{is}}=x_{i} p_{i}^{\text {sat }}$. Thus,

$$
y_{i}=\frac{p_{i}^{\text {sat }}}{p} x_{i}=K_{i} x_{i}
$$

This vapor-liquid equilibrium condition with $K_{i}=p_{i}^{\text {sat }} / p$ is known as Raoult's law. Raoult's law may be a good approximation for equilibrium calculations at low pressure.

\section{Algorithms for equilibrium calculations}

As indicated in Section 2, we need to be able to compute the vector field (the right hand side) of eqs. 1, 2, and 3. In addition, we want to compute $p, T$, and $\chi_{1}$ from $n_{1}$, $n_{2}$, and $U$. In order to do this, we first need to solve a PT flash. The PT flash requires us to compute the fugacity $f_{i}$ in each phase. 
When doing PT flash calculations, it is assumed that the contents of volume $V$ is in a two-phase state. Before performing the PT flash calculations, it should be checked whether this actually is true. Two phases are present if the pressure $p$ lies between pressures known as the bubble pressure $p_{\text {bubl }}$ and the dew pressure $p_{\text {dew }}$.

\subsection{PT flash}

As part of the PT flash calculation, we need to express $x_{i}$ and $y_{i}$ by $\left\{\chi_{i}\right\}$. This is done as follows. From Section 2 we have that $n_{i}=n_{i}^{L}+n_{i}^{V}$ and $n=n^{L}+n^{V}$. Furthermore, the mole fractions $x_{i}$ and $y_{i}$ sum up to unity. At equilibrium, we have found that $y_{i}=K_{i} x_{i}$. Thus,

$$
n_{i}=n_{i}^{L}+n_{i}^{V} \Leftrightarrow \chi_{i} n=x_{i} n^{L}+y_{i} n^{V}=x_{i}\left(n-n^{V}\right)+y_{i} n^{V} .
$$

By introducing the molar vapor fraction $v=n^{v} / n$, this leads to

$$
x_{i}=\frac{\chi_{i}}{1+v\left(K_{i}-1\right)} \text { and } y_{i}=K_{i} x_{i} \text {. }
$$

Next, introduce $F_{x y}(v) \triangleq \sum_{i=1}^{m} y_{i}-\sum_{i=1}^{m} x_{i}=0$. By inserting the expressions for $x_{i}$ and $y_{i}$, this yields

$$
F_{x y}(v)=\sum_{i=1}^{m} \frac{\chi_{i}\left(K_{i}-1\right)}{1+v\left(K_{i}-1\right)}=0 .
$$

It can be shown that $F_{x y}(v)$ is strictly decreasing in $v$, hence $F_{x y}(v)=0$ has a single real root. The root $v$ can be found by a Newton-Raphson search, by converting $F_{x y}(v)$ into a polynomial and using a polynomial solver, etc. In the case of a binary mixture, the solution of $F_{x y}(v)=0$ is

$$
v=\frac{\chi_{1}}{1-K_{2}}+\frac{\chi_{2}}{1-K_{1}}
$$

When Raoult's law is valid, $K_{i}=p_{i}^{\text {sat }} / p$ is independent of the compositions $\left\{y_{i}\right\}$ and $\left\{x_{i}\right\}$. In the binary case, the expressions for $v, x_{i}$, and $y_{i}$ thus become particularly simple.

For real mixtures with given/fixed $p$ and $T, K_{i}$ will be a function of the liquid composition $\left\{x_{i}\right\}$. Furthermore, since the real solution of $F_{x y}(v)=0$ also depends on $K_{i}$, it follows that $v$ varies with $\left\{x_{i}\right\}$. Thus, eq. 28 can in general not be solved explicitly with regards to $x_{i}$ since

$$
x_{i}=\frac{\chi_{i}}{1+v\left(\left\{x_{i}\right\}\right)\left(K_{i}\left(\left\{x_{i}\right\}\right)-1\right)} .
$$

It is thus necessary to introduce an iterative strategy in order to find $x_{i}$. Let $x_{i}^{j}$ denote iterative solution $j$ of $x_{i}$, and let $\left\{x_{i}^{j}\right\}$ denote $\left\{x_{1}^{j}, \ldots, x_{m}^{j}\right\}$. The simplest possible algorithm for solving such an implicit equation is the method of successive substitution (successive approximation) (Rice and Do, 1995; Hanna and Sandall, 1995), where we let

$$
x_{i}^{j}=\frac{\chi_{i}}{1+v\left(\left\{x_{i}^{j-1}\right\}\right)\left(K_{i}\left(\left\{x_{i}^{j-1}\right\}\right)-1\right)} .
$$

This is the algorithm proposed in, e.g., Smith and Van Ness (1987). More advanced methods for finding the roots of multivariable, nonlinear equations are discussed in, e.g., Lindfield and Penny (1995) and Dennis and Schnabel (1983). 


\subsection{Bubble point pressure and dew point pressure}

A convenient way of checking if a system is in a two-phase state is to calculate the bubble- and dew point pressures, and see whether the pressure of the system lies between these values. The bubble point pressure is the pressure where the first bubble is formed, while the pressure where the first droplet condenses out is termed the dew point pressure.

Consider the computation of the bubble point pressure $p_{\text {bubl. }}$. When the mixture is a subcooled liquid, $x_{i}=\chi_{i}$ at the given temperature $T$. Before the first bubble is formed, $y_{i}=0$. When the pressure $p$ decreases, the boiling point is approached. At the moment when the first vapor bubble is formed, $\sum_{i=1}^{m} y_{i}=1$. This pressure is defined to be $p_{\text {bubl }}$.

When Raoult's law is valid and with $x_{i}=\chi_{i}$,

$$
y_{i}=K_{i} x_{i}=x_{i} p_{i}^{\text {sat }} / p=\chi_{\mathrm{i}} p_{i}^{\text {sat }} / p .
$$

At the bubble pressure $p_{\text {bubl }}, \Sigma_{i=1}^{m} y_{i}=1$. Thus

$$
1=\sum_{i=1}^{m} \chi_{i} p_{i}^{\text {sat }} / p_{\text {bubl }} \Leftrightarrow p_{\text {bubl }}=\sum_{i=1}^{m} \chi_{i} p_{i}^{\text {sat }}
$$

For nonideal mixtures, $K_{i}$ varies with the composition $\left\{x_{i}\right\}$. The problem can then be posed as: find $p_{\text {publ }}$ such that $f^{V}\left(p_{\text {bubl }}, T,\left\{y_{i}\right\}\right)=f^{L}\left(p_{\text {bubl }}, T,\left\{\chi_{i}\right\}\right)$ with $\sum_{i=1}^{m} y_{i}=1$. When excess thermodynamics is used to describe $f_{i}^{L}$, this problem can easily be changed into a form suitable for the method of successive substitution (Smith and Van Ness, 1987). Since $f_{i}^{\text {ig }}$ is proportional to $p$, the proportional pressure dependence gets eliminated when using residual thermodynamics for both phases, and $f_{i}^{V}=f_{i}^{L}$ leads to $y_{i} \varphi_{i}^{V}\left(p_{\text {bubl }}, T,\left\{y_{i}\right\}\right)=\chi_{i} \varphi_{i}^{L}\left(p\right.$ bubl, $\left.T,\left\{\chi_{i}\right\}\right)$. The bubble point algorithm then gets more complicated (Smith and Van Ness, 1987).

The considerations and algorithms for computing the dew point pressure parallel those for computing the bubble point pressure.

\subsection{UV flash}

In the introduction, the UV flash problem is defined as calculating, e.g., $p, T$, and $\left\{\chi_{i}\right\}$ when $\left\{n_{i}\right\}$ and $U$ are known. Thus, we have the algebraic equations $\left\{n_{i}\right\}=f(p$, $\left.T,\left\{\chi_{i}\right\}\right)$ and $U=U\left(p, T,\left\{\chi_{i}\right\}\right)$. These equations usually have to be solved by iterations similar to those involved in the PT flash algorithm; in fact they involve PT flash calculations. The difficulty is to find good methods to update the estimated values of $p, T,\left\{\chi_{i}\right\}$. General methods for solving such problems are discussed in, e.g., Lindfield and Penny (1995), and Dennis and Schnabel (1983).

\section{Simulation example}

A one-stage $\mathrm{C}_{3}$ splitter has been considered, i.e., a flash tank with a mixture of propane and propylene.

First, a dynamic model was developed with equilibrium conditions based upon the Soave-Redlich-Kwong (SRK) equation of state. The resulting set of DAEs were implemented in the process simulator SPEEDUP (1993), which is an equation based simulator designed to solve DAEs. Sparse sets of equations are divided into blocks (block decomposition) which are then solved successively. The decomposition can not be manipulated by the user. The solution diverged, despite the correctness of the model. This was due to the block decomposition process. SPEEDUP's decomposition algorithm produced only two blocks of model equations, one of which contained only 

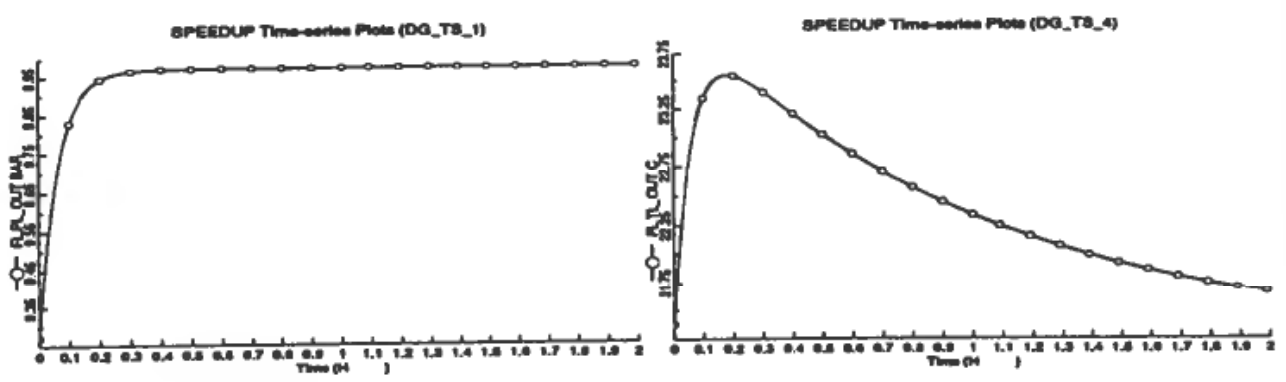

Figure 2. Time evolutions of pressure in bars (left) and temperature in centigrades (right).

one linear equation and one unknown, while the other block was made up of some thirty nonlinear equations in thirty unknowns.

Among many other routines, SPEEDUP has a routine for dynamic simulation of flash drums. Access to a data bank for physical properties of chemical species and FORTRAN procedures, where the thermodynamic calculations are executed, make SPEEDUP a suitable process simulator. A successful simulation for separation of propylene and propane was performed using the SPEEDUP routine FLASH. Figs. 2 and 3 show the time evolution of pressure, temperature, and liquid mole fractions in the propylene-propane system as calculated by SPEEDUP's flash routine.

Finally, a flash tank with propylene and propane was modeled using Raoult's equilibrium condition, and implemented in Matlab (Mathworks, 1992). Physical properties of these components are found in, e.g., Reid et al. (1988). The molar liquid volume was computed as the sum of the (constant) pure component molar liquid volumes, but weighted with the composition of the liquid mixture. The volume of the tank was $V=1 \mathrm{~m}^{3}$, the feed composition was $z_{1}=0.7$ (subscript 1 indicates propylene), the feed rate was $F^{F}=250 \mathrm{kmol} / \mathrm{h}$, and the molar feed enthalpy $\tilde{H}^{F}=-20 \cdot 4 \mathrm{~kJ} / \mathrm{mol}$. The flow of vapor out of the tank was $F^{V}=85 \mathrm{kmol} / \mathrm{h}$, and the added heat was $Q=5.46$ $\mathrm{kW}$. The initial values were $p=9.16 \mathrm{bar}, T=292 \mathrm{~K}$, and $\chi_{1}=0.7$. The process is perturbed by changing the liquid flow $F^{L}$ at initial time from $F^{L}=165 \mathrm{kmol} / \mathrm{h}$ to 149 $\mathrm{kmol} / \mathrm{h}$. These numbers differ from those used in the SPEEDUP simulations.

Fig. 4 displays the time evolution of this system.

The simulation results indicate that the temperature rapidly approaches the new steady state, while the composition changes slowly. The slow drift of the pressure is due to the slow changes in composition. As Fig. 4 indicates, $p$ approaches $p_{\text {bubl }}$ and
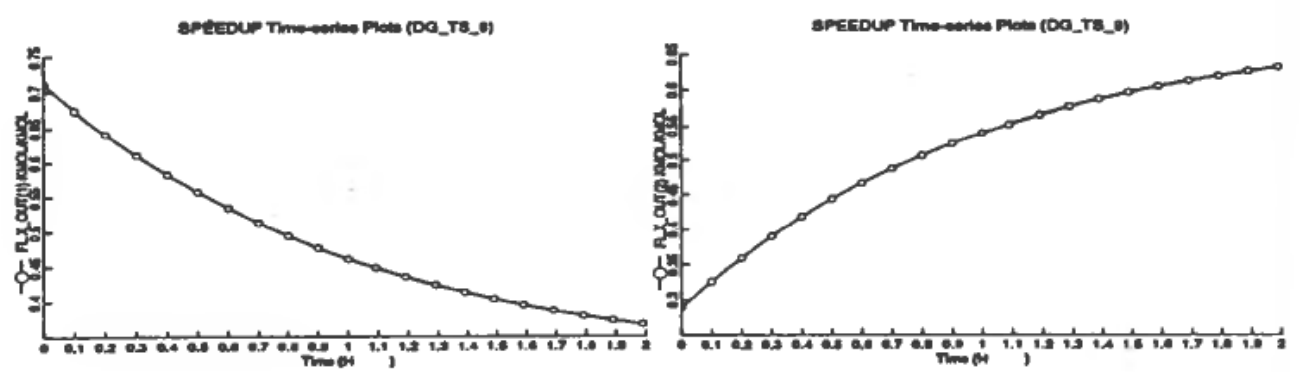

Figure 3. Time evolutions of liquid mole fractions of propane (left) and propylene (right). 

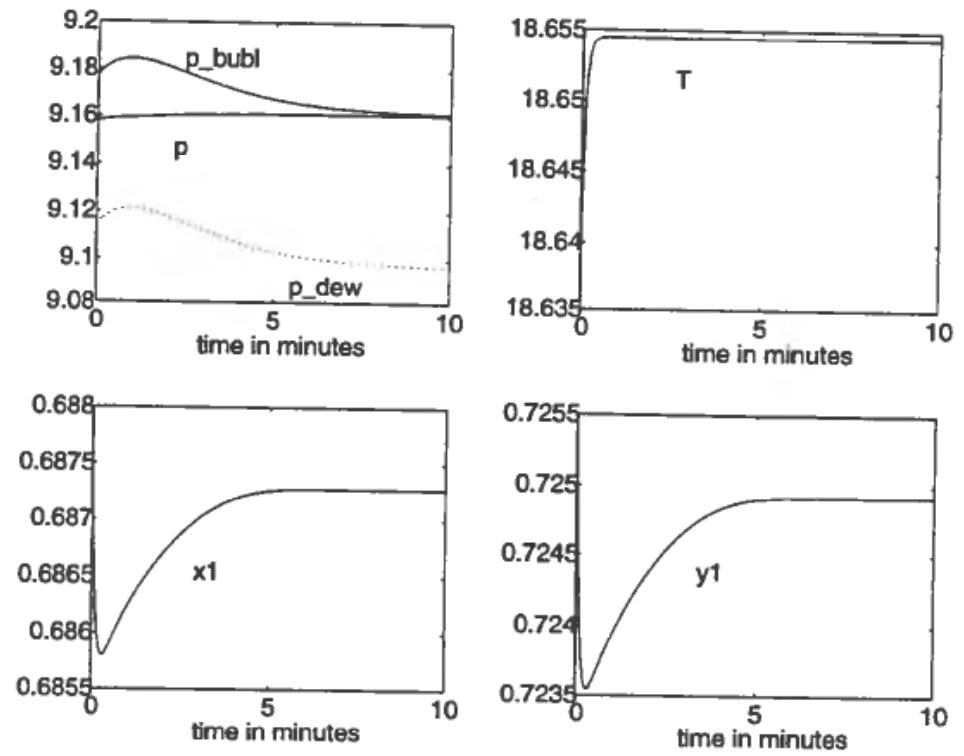

Figure 4. Time evolution of pressure $p$ (solid, marked p), bubble point pressure (solid, marked p_bubl), and dew point pressure (dotted, marked p_dew) in bars (upper left plot); temperature $T$ in ${ }^{\circ} \mathrm{C}$ (upper right plot); liquid mole fraction of propylene $x_{1}$ (lower left plot); and vapor mole fraction of propylene $y_{1}$ (lower right plot).

eventually the tank is filled with liquid. When the tank gets filled up, the number of states in the system is reduced by one, and $n_{1}+n_{2}$ becomes a constant.

\section{Conclusions}

This article gives an overview of modeling and simulation of dynamic systems with phase equilibrium. In general such systems are described by Differential-Algebraic Equations (DAEs). Several different strategies for solving the model equations are discussed. These strategies typically include PT flash and UV flash calculations, together with a check on whether the system is in a two-phase region or not. These computations are based upon thermodynamic models. To make the article more useful for those with less thermodynamic background, a brief survey of such models is given.

First, SPEEDUP was used to try to solve the DAEs with SRK based equilibrium conditions. The problem turned out to be too difficult for SPEEDUP to solve, and the equations never converged to a solution. The reason why SPEEDUP failed was the poor block decomposition of the DAEs. Next, the model was implemented in SPEEDUP using the built in FLASH tank module and the SRK based equilibrium conditions. This time, SPEEDUP solved the problem. These results illustrate the advantage of exploiting knowledge of the structure of the DAEs.

Finally, the dynamic model of a $\mathrm{C}_{3}$ flash tank with Raoult's law equilibrium conditions was implemented in Matlab to be able to more closely compare solution strategies. Propane and propylene have very close boiling points and are thus difficult to separate. It turned out to be rather difficult to tune initial values, etc. to make the model start in the two-phase region. Only minor changes were necessary in order to drive the system into a single phase state. This was caused by bubble- and dew point pressures that were sensitive to changes in the system. Also, due to the implemented model for 
the molar liquid volume, the system equations became stiff when the tank was (almost) filled up with liquid.

The DAEs were solved using $p, T$, and $\chi_{1}$ as states; simulation results are reported in the Simulation example section. The UV flash calculation was eliminated by numerically computing the Jacobian $J$ between coordinates $\left(p, T, \chi_{1}\right)$ and $\left(n_{1}, n_{2}, U\right)$. Unless the model equations were properly scaled, the Jacobian became poorly conditioned.

The DAEs were also simulated using an optimization code to solve the UV flash problem. Both the leastsq routine of the Matlab Optimization Toolbox, MathWorks (1990), and routine e04jaf of the NAG Foundation Toolbox, MathWorks (1995), were used in the UV flash calculations. It turned out to be important to scale the model equations to speed up convergence. Preliminary experience indicates that it is important to use industrial quality optimization code. It is also our experience that the simulation takes more time when rigorous UV flash calculations are used.

In practical process simulations, the thermodynamic calculations are often responsible for more than $90 \%$ of the simulation time. It is thus necessary to use efficient numerical algorithms for solving the equilibrium conditions. The simulation examples reported in this article also illustrate the necessity of using high quality numerical algorithms. Numerical algorithms for solving thermodynamic problems are discussed in, e.g., Smith and Missen (1982).

\section{REFERENCES}

BeJAN, A. (1988). Advanced Engineering Thermodynamics. John Wiley \& Sons, New York. BOSLEY, J. R., JR. (1994). An Experimental Investigation of Modeling, Control, and Optimization Techniques for Batch Distillation. Ph.D. dissertation, the University of Texas, Austin.

BRENAN, K. E., CAMPBELL, S. L. and PETZOLD, L. R. (1989). Numerical Solution of Initial-Value Problems in Differential-Algebraic Equations. North-Holland, New York.

CALLEN, H. B. (1985). Thermodynamics and an introduction to Thermostatistics, second edition. John Wiley \& Sons, New York. First edition: 1960.

Char, B. W., Geddes, K. O., GonNet, G. H., Leong, B. L., Monagan, M. B., and WatT, S. M. (1992). First Leaves: A Tutorial Introduction to Maple V. Springer-Verlag, New York.

DenN, M. (1987) Process Modeling. Longman Scientific \& Technical, Harlow.

DENNIS, J. E., JR. and SCHNABEL, R. B. (1983). Numerical Methods for Unconstrained Optimization and Nonlinear Equations. Prentice-Hall, Englewood Cliffs, NJ. Reprint (1996) in series Classics In Applied Mathematics No. 16, SIAM, Philadelphia.

Gani, R., PERrEgaARD, J. and Johansen, H. (1990). Simulation Strategies for Design and Analysis of Complex Chemical Processes. Trans. Inst. Chem. Eng., pp. 407-417.

GILl, P. E., MURRAY, W. and WrIGHT, M. H. (1981). Practical Optimization. Academic Press, London.

GmeHLING, J. and KolBE, B. (1988). Thermodynamik. (In German.) Georg Thieme Verlag, Stuttgart.

GunDERSEN, T. (1982). Numerical Aspects of the Implementation of Cubic Equations of State in Flash Calculations. Comp. Chem. Eng., Vol. 6, p. 245.

Hanna, O. T. and Sandale, O. C. (1995). Computational Methods in Chemical Engineering. Prentice-Hall, Upper Saddle River, NJ.

HYSYS (1995). From: HYPROTECH, Calgary, Canada.

LindFIELD, G. and PENNY, J. (1995). Numerical Methods Using Matlab. Ellis Horwood, New York.

MATHWORKS (1990). Optimization Toolbox for use with MATLAB. The MathWorks, Inc., Natick, Massachusetts.

MATHWORKS (1992). MATLAB User's Guide. The MathWorks, Inc., Natick, Massachusetts. 
MATHWORKS (1995). NAG Foundation Toolbox for use with MATLAB. The MathWorks, Inc., Natick, Massachusetts.

Modell, M. and ReID, R. C. (1983). Thermodynamics and Its Applications, second edition. Prentice-Hall, Inc., Englewood Cliffs, NJ. First edition: 1974.

MoE, H. I. (1995). Dynamic Process Simulation. Studies on modelling and index reduction. Dr.ing. dissertation, The Norwegian Institute of Technology, Trondheim.

Prausnitz, J. M., Lichtenthaler, R. N. and DE Azevedo, E. G. (1986). Molecular Thermodynamics of Fluid-Phase Equilibria, second edition. Prentice-Hall, Inc., Englewood Cliffs, NJ.

RAmireZ, W. F. (1989). Computational Methods for Process Simulation. Butterworths, Boston.

Reid, R. C., Prausnitz, J. M. and Poling, B. E. (1988). The Properties of Gases and Liquids, 4th edition. McGraw-Hill Book Company, New York.

RICE, R. G. and Do, D. D. (1995). Applied Mathematics and Modeling for Chemical Engineers. John Wiley \& Sons, Inc., New York.

SANDLER, S. I. (1989). Chemical and Engineering Thermodynamics, second edition. John Wiley \& Sons, New York.

SANDLER, S. I. (1994). Models for Thermodynamic and Phase Equilibria Calculations. Marcel Dekker, New York.

SMith, W. R. and MisSEN, R. W. (1982). Chemical Reaction Equilibrium Analysis: Theory and Algorithms. John Wiley \& Sons, New York.

SMITH, J. M. and VAN NESS, H. C. (1987). Introduction to Chemical Engineering Thermodynamics. Fourth Edition. McGraw-Hill Book Company, New York.

SPEEDUP (1993). Speedup-Systems Manual. Aspen Technology, Inc., Cambridge, Massachusetts. 